\title{
Laboreal
}

Volume $14 \mathrm{~N}^{\circ} 2$ | 2018

Digitalização e evolução do trabalho real

\section{Comprender la actividad de diseño y su desarrollo}

Compreender a atividade de conceção e o seu desenvolvimento

Comprendre l'activité de conception et son développement

Understanding the design activity and its development

\section{Annie Weill-Fassina}

\section{OpenEdition}

\section{Journals}

Edición electrónica

URL: http://journals.openedition.org/laboreal/811

DOI: 10.4000/laboreal.811

ISSN: 1646-5237

\section{Editor}

Universidade do Porto

\section{Referencia electrónica}

Annie Weill-Fassina, "Comprender la actividad de diseño y su desarrollo », Laboreal [En línea], Volume $14 N^{0} 2$ | 2018, Publicado el 01 diciembre 2018, consultado el 24 septiembre 2020. URL : http:// journals.openedition.org/laboreal/811 ; DOI : https://doi.org/10.4000/laboreal.811

Este documento fue generado automáticamente el 24 septiembre 2020

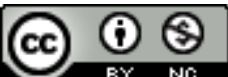

Laboreal está licenciado com uma Licença Creative Commons - Atribuição-NãoComercial 4.0 Internacional. 


\title{
Comprender la actividad de diseño y su desarrollo
}

\author{
Compreender a atividade de conceção e o seu desenvolvimento \\ Comprendre l'activité de conception et son développement \\ Understanding the design activity and its development
}

Annie Weill-Fassina

\section{REFERENCIA}

Comentario del texto de Lebahar, J.-Ch. (2007). La conception en design industriel et en architecture. Désir, pertinence, coopération et cognition (pp. 15-21). Paris: HermesLavoisier.

\section{NOTA DEL EDITOR}

http://dx.doi.org/10.15667/laborealxiv218awf

Manuscrito recibido en: Junio / 2018

Aceptado tras peritaje: Septiembre /2018

1 En la introducción del libro 'La conception en design industriel et en architecture ${ }^{\iota_{1}}$, publicado en 2007, Jean-Charles Lebahar especifica su perspectiva sobre la diseño: "la actividad de diseño es la construcción y la comunicación de un modelo de artefacto que no resulta de la réplica de un modelo (pre)existente' (p. 15), debiendo la elaboración de este modelo permitir la realización del artefacto por aquellos que tendrán a su cargo su fabricación. También especifica su intención: 'Los análisis de actividad recogidos en este libro bajo la forma de estudios de caso se centran en un sujeto psicológico que concibe artefactos, o sea 'un sujeto diseñador' (idem, p. 15). En la situación de diseño, intervienen en simultáneo las características del sujeto diseñador y las de la tarea (restricciones técnicas y 
operacionales). 'El enfoque interdisciplinar permite definir la actividad del sujeto diseñador bajo un ángulo cognitivo y semiológico en simultaneo' (p. 18).

\section{La diseño no es una resolución de un problema mal definido}

2 Aunque no sea él mismo el productor del artefacto, el diseñador debe conocer e integrar en su modelo las técnicas y restricciones de fabricación o de construcción para que la realización sea posible. Desde el punto de vista cognitivo, se constata pues desde el inicio del proceso de diseño que, para el diseñador, (como es, de hecho, frecuente en ergonomía) se trata más de construir una representación de la situación de diseño que deberá transformar, que sólo plantear y resolver un problema mal definido.

De este análisis, Lebahar concluye que el diseño no es identificable con la resolución de un problema. Para él, as diferencias encuentran fundamento en distintas constataciones:

- La formulación del problema y, por lo tanto, la tarea de diseño, evoluciona de manera imprevista en el transcurso de la actividad, lo que da a esa actividad un avance oportunista.

- La situación de diseño no consiste en un solo problema, pero en distintos problemas con múltiples formas y múltiples dominios que requieren una articulación entre ellos;

- La actividad no es reductible a un código único de acciones y operaciones como en una demostración matemática o en procedimientos como es el caso de la ‘torre de Hanói’ o del 'juego de Marienbad' ';

- Los procedimientos, como los resultados, varían de un diseñador a otro;

- Las soluciones elegidas se consideran satisfactorias según un determinado número de criterios, pero raras veces son óptimas.

Según el autor,

5 'se puede optar por la reducción de la situación a la resolución un problema mal definido efectuada por un sistema de procesamiento de información. Se puede, em cambio, como es aquí el caso, considerar que una tarea de diseño se sitúa en un entorno histórico de restricciones y de recursos, y que esa tarea es realizada mediante una competencia bastante más compleja que un sencillo sistema de procesamiento de la información. La competencia del diseño no puede ser reducida a un autómata informático cuyas operaciones obedecen a sencillas reglas y definiciones de un código de interpretación de las conductas humanas aplicables a priori e indistintamente a tareas tan complejas como el diseño de la carrocería de un automóvil, de un edificio o de una linterna de bolsillo. La competencia de un sujeto diseñador encierra, sin reducirse a ello, un sistema de procesamiento de información. Pero esa competencia está también hecha de sistemas de valores, de creencias y de significados que se encuentran en el origen de las hipótesis de diseño que el sujeto elabora o que toma prestado de otras personas' (2007, p. 32).

Si el trabajo de diseño puede llevar a pensar en el trabajo de creación, se diferencia de éste en numerosos aspectos.

\section{Diseño y creación, actividades distintas}

7 En lo que respecta a las relaciones entre diseño y creatividad, Lebahar asevera con claridad que 'el sujeto diseñador no podría realizar su tarea si se encontrase en un desierto 
social y técnico, contrariamente a la mitología del acto creador'. De hecho, la descripción del diseño arquitectural y de los procesos de diseño industrial no se basa 'en una cadena de reacciones totalmente subjetivas. La lucha por la realización es una sucesión de esfuerzos, de dolores, de satisfacciones, de rehúsas, de decisiones que no pueden ni deben ser plenamente conscientes, por lo menos en el plano estético' (2007, p. 30).

8 Si la evolución de las representaciones puede hacer pensar por momentos a un acto creador, los análisis subrayan características específicas del diseño:

- un objeto dirigido desde el exterior;

- un objeto no solo estético, sino también funcional y seguro;

- la ausencia de creación ex-nihilo;

- la dependencia o la sumisión a restricciones ante las cuales es necesario responder.

9 Esta diferenciación del 'acto creador' no dice todavía nada sobre la 'creatividad' del diseñador. Se puede definir la creatividad como 'la capacidad de un individuo o de un grupo de imaginar o construir y poner en práctica un concepto nuevo, un objeto nuevo o de descubrir una solución original a un problema's. A este nivel muy global, no se puede negar que la noción de creatividad, aunque no se sepa ni 'evaluarla' ni 'medirla', se puede aplicar a ciertos diseñadores en arquitectura o en diseño en virtud de la originalidad de sus proyectos o de su realización. Pero el imaginario no tiene la misma función en el diseño y en el arte. Como nota Lebahar en una de sus obras precedentes,

10 'la creación en el arte no es comparable a las otras actividades humanas de diseño aunque se trate de crear objetos artificiales que fascinen a sus espectadores. El imaginario y el fantasma son sus motores evidentes, pero les proporcionan esencialmente trámites, energías y estilos que, bajo la forma de deseos y de esquemas, modelan su valor de uso. En el arte, el imaginario y el fantasma son maneras de ver el mundo que suponen también verse a sí mismo y mostrarse al otro.' (1998 p.12-13).

11 Es por esto que, además de estas primeras definiciones, 'el proceso creativo y el proceso de diseño' no pueden ser asimilados. En general, el proceso creativo es definido como 'un proceso mental que involucra la generación de nuevas ideas o conceptos o nuevas asociaciones entre ideas y conceptos preexistentes's. Lebahar se apoya en John-Laird para poner en duda

'esa creencia ingenua según la cual la creación resultaría sistemáticamente de asociaciones de ideas que no son habitualmente asociadas. Ese punto de vista crítico se opone a la tesis mecanicista de la 'analogía creativa', el hecho de que cualquier creación de una novedad presupone el respecto por ciertas restricciones y la transformación en obra de conocimientos profundos tal como los procesos de elaboración de hipótesis de diseño que no siempre son instantáneos' (2007, p. 89).

13 Para Lebahar (2008), el diseñador es el heredero del progreso técnico y de sus consecuencias organizacionales, dependiendo sus realizaciones de las evoluciones de las situaciones y de su propia evolución. Distintas observaciones llevan a subrayar la influencia de la herramienta, y en particular del ordenador, en la exploración de las posibilidades de soluciones alternativas y el control de las representaciones intermediarias, así como la influencia del conocimiento de los materiales disponibles para adaptar mejor las distintas posibilidades de artefactos a lo solicitado. Si no se considera nada más que los extremos, podría decirse que si la familiaridad, en particular con el ordenador, puede inducir distintas estrategias de diseño, ciertos artistas buscan ante todo apropiarse y probar distintas técnicas para obtener los 
resultados que desean. Un punto esencial del proceso de diseño persiste: 'una particular atención al control, puesto que algunas de sus inspiraciones espontáneas son susceptibles de provocar catástrofes, mientras que 'relaciones impulsivas, rápidas, fulgurantes, happenings...' (Lebahar, 1988 p. 12) soportan ciertas corrientes artísticas. Un diseñador reputado no reducirá la incertidumbre demasiado rápido 'para no ser sorprendido, porque los errores cuestan caro'. La función de los modelos de simulación es en simultáneo instrumento de representación, de control y de regulación de la actividad de diseño, ya sea esta individual o colectiva. (Lebahar, 1997).

Esta oposición, sin embargo, debe ser matizada: parece que cuanto más nos acercamos a la técnica más se desarrollan procesos de ajuste y de autocontrol en la creación artística antes de crear la obra definitiva. Rodin creaba sus estatuas en yeso antes de la confección de los moldes donde era fundido el bronce. Heinrich Khün hacía bocetos de sus paisajes y sometía sus modelos a interminables sesiones de pose antes de hacer la fotografía. Estudiaba los gradientes de luz y de luminosidad, experimentando distintas técnicas de impresión.

El término inventividad, que tiene una resonancia más técnica y científica, correspondería más al diseño arquitectural y al diseño que a la de creatividad que induce ante todo a la idea de estética, de arte, aunque los aspectos estéticos tengan un lugar importante en la diseño. 'Un producto que no sea bonito no se compra y un producto funcionalmente perfecto tampoco se compra si un producto de la competencia es más bonito' decía un diseñador que Lebahar observó (1996, p. 128), pero para este se trata más de ‘seducción' que de estética (idem, p. 131).

\section{El desarrollo de competencias en diseño como equilibrio}

Esta caracterización de los procesos de diseño hace sobresalir la extrema complejidad de esta actividad que presupone conocimientos, saberes y saber haceres en multidominios, a poner en práctica en situaciones operacionales, ellas mismas complejas. Los indicadores de competencia, sobre todo los cualitativos, son numerosos y pueden ser oriundos tanto de observables de actividad (calidad y valor de utilización del producto, dominio de las restricciones y recursos del entorno) como inferidos de la observación del desarrollo de esta actividad y de entrevistas (conocimientos, metaconocimientos, esquemas de acción, etc., modalidades de autorregulación de la actividad y de regulación del entorno profesional (Lebahar, 1997 b).

17 En términos generales, Lebahar asimila la producción de un modelo de artefacto a una microgénesis. Apoyándose en la teoría del equilibrio de Piaget describe, en un marco constructivista, la elaboración de modelos entre el arte y la técnica para responder a las restricciones materiales de la situación y elaborar compromisos entre las exigencias de los distintos actores. Desde una perspectiva piagetiana, que es la suya, 'el enfoque cognitivo del proceso de diseño se basa en la hipótesis de un equilibrio entre el sistema humano de producción de conocimientos del diseñador y la solución del problema del diseño, la representación final del producto. (Lebahar, 1993, p.39). El análisis de la evolución de las representaciones intermediarias producidas por los arquitectos y diseñadores reputados permiten a Lebahar mostrar que su actividad de equilibrio se desdobla en dos mecanismos: por un lado, construye soluciones que él mismo controla, evalúa y 
conserva o rechaza en la investigación constructiva de una solución sintética, el producto global. Al mismo tiempo, Lebahar insiste en el hecho de que actúa sobre esta construcción de soluciones, 'su solución', para garantizar su éxito, pese a que la modifica parcialmente cuando el obstáculo en cuestión es una restricción que se revela ineludible. Este desdoblamiento cognitivo surge como una característica esencial de la actividad del diseñador.

En un intento de comprender la génesis de la pericia a más largo plazo, Lebahar compara la actividad de un diseñador CAD reputado con la de un novato, 'encargado de establecer modelos geométricos simulando las sucesivas hipótesis de un puesto de pilotaje'. Siempre apoyando su hipótesis general en la teoría de la equilibrio, sostiene que 'la adquisición de las capacidades operacionales equivalentes a las de un diseñador CAD experimentado - recuperar las informaciones, utilizar un dominio de conocimiento amplio $y$ estructurado, controlar su propia actividad - resulta del desarrollo de la pericia de un novato y de los intentos de control que éste aplica a ese desarrollo' (1997 C). El análisis de la actividad del diseñador $C A D$ novato pone efectivamente en evidencia un relativo desconocimiento de los objetos del terreno, una organización fragmentada de los objetos y de los medios operativos que le corresponden, la utilización de trazos superficiales a los cuales es difícil hacer corresponder un significado, dificultades de pasar del dibujo 2D a una interpretación en 3D, investigaciones prematuras de significado, analogías que no son necesariamente pertinentes; encontramos aquí numerosas características comunes al desarrollo de competencias profesionales a partir de la experiencia como ya pudieron ser puestas en evidencia en otros oficios (WeillFassina \& Pastré, 2004).

El análisis de la actividad de los estudiantes-diseñadores en situación pedagógica permite retroceder todavía más en la génesis de las competencias en diseño. En esta etapa, 'la situación permite poner en evidencia indicadores de competencia de diseño, pero también conductas de asimilación subjetiva' tal como las definía Piaget (1968). Se entenderán como 'asimilación subjetiva' las conductas que, para un sujeto diseñador, consisten en adaptar consciente o inconscientemente una tarea de diseño a sus propios objetivos sin que estos no sean objeto de una necesaria acomodación a las restricciones de esa tarea' (Lebahar 2007, p. 244).

Estos análisis muy finos del desarrollo de las actividades de diseño desde una perspectiva micro y macrogenética permiten hacer frente a una enseñanza del diseño industrial que no es tanto una enseñanza de la creatividad como una metodología de la diseño apoyada en múltiples conocimientos pluridisciplinares. Es este punto de vista didáctico que desarrolla en su última obra L'enseignement du design industriel ${ }^{\prime 5}$ surgida en 2008, dos años antes de su fallecimiento en 2010.

\section{BIBLIOGRAFÍA}

Lebahar, J.C. (1993) Aspects cognitifs du travail de designer industriel. Design recherche, 3, 39-55. 
Lebahar, J.C. (1996) Séduction. in Dictionnaire international du graphisme et du design industriel. Paris: Éditions du Regard.

Lebahar, J.C. (1997 a) La simulation, instrument de représentation et de régulation dans la conception de produit. In P. Béguin et A. Weill-fassina (Eds), La simulation en ergonomie [77-96]. Toulouse: Octares.

Lebahar, J.C.(1997 b) Complexité des compétences de création industrielle: Qui évalue? Quoi? Comment? Connexions, 70, 151-164.

Lebahar, J.C. (1997 c) Genèse d'une expertise: Le cas d'un dessinateur novice destiné à succéder à un expert en simulation CAD. Tapuscrit, 25 pages. Communication personnelle)

Lebahar, J.C. (1998) Christian Jaccard. Montreuil-sous-Bois: Au même titre éditions

Lebahar, J.C. (2007) La conception en design industriel et en architecture. Paris: Hermés, Lavoisier.

Lebahar, J.C. (2008) L'enseignement du Design industriel. Paris: Hermes, Lavoisier.

Piaget, J. (1968) La formation du symbole chez l'enfant. Lausanne: Delachaux et Nestlé.

Weill-Fassina, A. \& Pastré, P. (2004) Les compétences professionnelles et leur développement.

\section{NOTAS}

1. Nota del traductor: "La concepción en el diseño industrial y en la arquitectura".

2. Nota del traductor: La descripción sumaria de estos juegos puede ser encontrada en https:// es.wikipedia.org/wiki/Torre_de_Hanói e https://en.wikipedia.org/wiki/ Marienbad_(video_game).

3. Nota del traductor: traducida desde el francés: https://fr.wikipedia.org/wiki/Créativité.

4. Nota del traductor: traducida desde el francés: https://fr.wikipedia.org/wiki/Créativité.

5. Nota del traductor: La enseñanza del diseño industrial.

\section{AUTOR}

\section{ANNIE WEILL-FASSINA}

GRESHTO - CRTD - CNAM

41 Rue Gay-Lussac 75005 Paris, France

weill.fassina@orange.fr 